\title{
Examples in the Geometry of Crose Ratios
}

\section{By Charles Tweedie}

(Read 8th May. MS. received 2nd June, 1908)

When $\mathrm{P}$ is joined to four points $\mathrm{A}, \mathrm{B}, \mathrm{C}, \mathrm{D}$ coplanar with $\mathrm{P}$, a pencil of four lines is formed whose cross ratio is constant if $\mathrm{ABCD}$ are collinear. If $\mathrm{A}, \mathrm{B}, \mathrm{C}, \mathrm{D}$ are not in a line the cross ratio $P(A B C D)$ has a value which in general varies with the position of $P$, but which should be known when $P$ is given in position and also A, B, C, D. A simple expression for the cross ratio is given and its utility in locus problems is illustrated by a variety of simple examples, which in several cases furnish methods for constructing a general cubic curve, with or without double point, a trinodal quartic, etc.

$\$ 1$. Let $1,2,3,4$ be four points in a plane, $P$ any fifth point. There exists the following relation in the signed areas of the triangles $\mathrm{P} 12$, etc.

$$
(\mathrm{P} 12)(\mathrm{P} 34)+(\mathrm{P} 13)(\mathrm{P} 42)+(\mathrm{P} 14)(\mathrm{P} 23)=0 \quad-\quad-\quad \mathrm{I} \text {; }
$$

and the cross ratio of the pencil $P(1234)$ is given by

$$
\mathrm{P}(1234)=(\mathrm{P} 13)(\mathrm{P} 24) /(\mathrm{P} 14)(\mathrm{P} 23)-\quad-\quad-\quad-\quad I I .
$$

These are easily established. Let $|\mathrm{P} 1|=r_{1},|\mathrm{P} 2|=r_{2}$, etc. ; and let (12) denote the signed angle $1 \mathrm{P2}$.

Then $\mathrm{P} 12=\frac{1}{2} r_{1} r_{2} \sin (12) ; \mathrm{P} 34=\frac{1}{2} r_{3} r_{4} \sin (34)$; etc.

Substitution in $\mathrm{I}$ leads to the trigonometrical identity $\sin (12) \sin (34)+\sin (13) \quad \sin (24)+\sin (14) \quad \sin (23)=0$ and the right side of II reduces to the well-known expression for the cross ratio $\sin (13) \sin (24) / \sin (14) \sin (23)$.

Cor. 1. $\mathrm{P}(1234)=\mathrm{P}(1235) . \mathrm{P}(1254)$;

$$
=(\mathrm{P} 1235) \mathrm{P}(1256) \mathrm{P}(1264) ; \text { etc. }
$$

Cor. 2. $\mathrm{P}(1234)$. $\mathrm{P}(1342) \mathrm{P}(1423)=-1$.

Cor. 3. $\mathrm{A}(\mathrm{BCPQ})$. B(CAPQ) $\mathrm{C}(\mathrm{ABPQ})=+1$.

Cor. 4. Divide in $\mathrm{I}$ by (P14) (P23) when it gives rise to $\mathrm{P}(1234)+\mathrm{P}(1324)=1$. 
\$2. Let $\mathrm{P}(1234)=\lambda$ it is then easy to establish the following well-known relations either by direct substitution in II or by the aid of Cor. 1. and Cor. 4. of $\$(1)$ :

$$
\begin{aligned}
& \mathrm{P}(1234)=\mathrm{P}(2143)=\mathrm{P}(3412)=\mathrm{P}(4321)=\lambda \\
& \mathrm{P}(1243)=1 / \lambda, \\
& \mathrm{P}(1324)=1-\lambda, \\
& \mathrm{P}(1342)=1 /(1-\lambda), \\
& \mathrm{P}(1423)=1-1 / \lambda=(\lambda-1) / \lambda, \\
& \mathrm{P}(1432)=\lambda /(\lambda-1) .
\end{aligned}
$$

$\S 3$. In the dual problem let 1234 be a transversal to the sides of a quadrilateral ABCD as in Figure 7.

Let $a, b, c, d$ be the perpendicular distances of the vertices of $\mathrm{ABCD}$ from the transversal.

Denote by $\overline{13}$ the directed segment from 1 to 3 , and by (13) the angle between the sides of the quadrilateral that pass through 1 and 3 . Let $\sin (1234)$ denote $\sin (13) \sin (24) / \sin (14) \sin (23)$.

Then

$$
\begin{aligned}
& \mathrm{A} 1 . \mathrm{A} 3 \sin (13)=a \cdot \overline{13}=2 \triangle \mathrm{A} 13 \\
& \mathrm{C} 2 . \mathrm{C} 4 \sin (24)=c \cdot \overline{24}=2 \Delta \mathrm{C} 24 \\
& \mathrm{~B} 1 . \mathrm{B} 4 \sin (14)=b \cdot \overline{14}=2 \triangle \mathrm{B} 14 \\
& \mathrm{D} 2 . \mathrm{D} 3 \sin (23)=d \cdot \overline{23}=2 \triangle \mathrm{D} 23 .
\end{aligned}
$$

Hence

$$
\begin{aligned}
& \frac{\mathrm{A} 1}{\mathrm{~B} 1} \cdot \frac{\mathrm{C} 2}{\mathrm{D} 2} \cdot \frac{\mathrm{A} 3}{\mathrm{D} 3} \cdot \frac{\mathrm{C} 4}{\mathrm{~B} 4} \times \sin (1234) \\
& =\frac{a c}{b d}(1234)
\end{aligned}
$$

i.e. $\quad \frac{a c}{b d} \sin (1234)=(1234)\left(\because \frac{\mathrm{A} 1}{\mathrm{~B} 1}=\frac{a}{b} ;\right.$ etc $)$

Also

$$
\begin{gathered}
(\mathrm{A} 13)(\mathrm{C} 24) /(\mathrm{B} 14)(\mathrm{D} 23)=\frac{a c}{b d}(1234) \\
\therefore \frac{\mathrm{A} 13 \cdot \mathrm{C} 24}{\mathrm{~B} 14 \cdot \mathrm{D} 23} \sin (1234)=(1234)^{2}
\end{gathered}
$$

Cor. From these equations a great variety of identities may be deduced. In particular if $(1234)=-1$ we obtain 


$$
\frac{(1 \mathrm{~A} 3)(2 \mathrm{C} 4)}{(1 \mathrm{~B} 4)(2 \mathrm{D} 3)} \times \frac{\sin (1 \mathrm{~A} 3) \sin (2 \mathrm{C} 4)}{\sin (1 \mathrm{~B} 4) \sin (2 \mathrm{D} 3)}=1 \text {. }
$$

Similarly when ABCD are united into a single point we obtain the formula in areas given in $\$(1)$.

$\$ 4$. If $\mathrm{P}, 1,2,3,4$ have co-ordinates $(x y z) ;\left(x_{1} y_{1} z_{1}\right)$; etc., then

$$
P(1234)=\frac{\left(x y_{1} z_{3}\right)\left(x y_{2} z_{4}\right)}{\left(x y_{1} z_{4}\right)\left(x y_{2} z_{3}\right)}
$$

where $\left(x y_{1} z_{3}\right)$ is the determinant whose rows are $x y z ; x_{1} y_{1} z_{1} ; x_{3} y_{3} z_{3}$.

For non-homogeneous co-ordinates put $z=1$ throughout.

In the dual theorem if 1234 is the line $l x+m y+n=0$ and the lines through $1,2,3,4$ forming the sides of $\mathrm{ABCD}$ are $l_{1} x+m_{1} y+n_{1} z=0$, etc., then

$$
(1234)=\frac{\left(l m_{1} n_{3}\right)\left(l m_{2} n_{4}\right)}{\left(l m_{1} n_{4}\right)\left(l m_{2} n_{3}\right)} .
$$

For non-homogeneous co-ordinates put $n=1$ throughout. The identity of form in these two analytical expressions enables us to dispense with the distinction of co-ordinates.

$\S 5$. If five points $1,2,3,4,5$ are taken and $P$ be any other point in their plane, there are but two independent cross ratios formed by joining $\mathbf{P}$ to four of these points.

$$
\begin{aligned}
& \text { Let } \mathbf{P}(\mathbf{1 2 3 4})=\lambda \text {; } \quad-\quad-\quad-\quad-\quad-\quad \text { - } \mathbf{I} \\
& \mathrm{P}(1235)=\mu \quad-\quad-\quad-\quad-\quad-\quad \text { - II } \\
& \text { Then } P(1254)=P(1234) / P(1235)=\lambda / \mu \quad-\quad-\quad \text { III } \\
& \mathrm{P}(1345)=\mathrm{P}(1325) / \mathrm{P}(1324)=(1-\mu)(/ 1-\lambda) \quad \text { IV } \\
& \mathrm{P}(2345)=\mathrm{P}(2315) / \mathrm{P}(2314) \\
& =\lambda(\mu-1) / \mu(\lambda-1) \quad-\quad-\quad \mathrm{V}
\end{aligned}
$$

It therefore follows that if abcd are any four of the points 12345 then $\mathrm{P}(a b c d)$ is a rational function of $\lambda$ and $\mu$ linear in either of the variables. The elimination of $\lambda$ and $\mu$ from these relations gives rise to a great variety of identical relations.

Cor. The equations I-V also furnish the values of the cross ratio of any four of the five points 1, 2, 3, 4, 5 for the conic containing all the five points. 
These may also be verified for the conic as follows. We have the identity

\begin{tabular}{|c|c|}
\hline & $5(1234) \cdot 3(1245) \cdot 4(1253)=1$ \\
\hline \multirow[t]{2}{*}{ If } & $\lambda=5(1234)$ \\
\hline & $\mu=4(1235)$ as on the conic \\
\hline then & $3(1245) \times \lambda \times 1 / \mu=1$ \\
\hline Hence & $3(1245)=\mu / \lambda$ \\
\hline & $3(1254)=\lambda / \mu$ as in III. \\
\hline
\end{tabular}

$\S 6$. If $\mathrm{P}(1234)=\lambda ; \mathrm{P}(1235)=\mu$, then $\mathrm{P}$ is thereby uniquely determined. For $P(1234)=\lambda$ represents a conic through $1,2,3,4$; and $\mathrm{P}(1235)=\mu$ a second conic through $1,2,3,5$. The conics cut in $1,2,3$ and in the unique point $P$. We may therefore speak of $\lambda$ and $\mu$ as being the co-ordinates of $P$. Only when $\mathrm{P}(1234)=5(1234) ;$ and $\mathrm{P}(1235)=4(1235)$ is $\mathrm{P}$ indeterminate, being then any point on the conic through $1,2,3,4,5$.

The expression of $\lambda$ and $\mu$ in trilinear co-ordinates may be conveniently found by taking $1,2,3$ as triangle of reference. Let 4 be the point $(\alpha \beta \gamma), \mathrm{P}$ the point $(x y z)$.

By the data

$$
\lambda=\Delta \mathrm{P} 13 \cdot \Delta \mathrm{P} 24 / \triangle \mathrm{P} 14 \cdot \Delta \mathrm{P} 23 ;
$$

and on making the calculation we find

$$
\lambda=(\gamma / z-\alpha / x) /(\gamma / z-\beta / y) \quad \text {. . . }
$$

Similarly if 5 is the point $\left(a^{\prime}, \beta^{\prime}, \gamma^{\prime}\right)$

$$
\mu=\left(\gamma^{\prime} / z-a^{\prime} / x\right) /\left(\gamma^{\prime} / z-\beta^{\prime} / y\right)
$$

Equations (1) and (2) furnish the relations

$$
\begin{aligned}
& \frac{1}{x}: \frac{1}{y}: \frac{1}{z}=\lambda \mu\left(\beta^{\prime} \gamma-\beta \gamma^{\prime}\right)-\beta^{\prime} \gamma \mu+\beta \gamma^{\prime} \lambda \\
& : a^{\prime} \gamma(\lambda-1)-\alpha \gamma^{\prime}(\mu-1): \alpha^{\prime} \beta \lambda-a \beta^{\prime} \mu .
\end{aligned}
$$

Cor. The equation to a line being of the form $\mathrm{A} / y z+\mathrm{B} / z x+\mathrm{C} / x y=0$, it follows that to a straight line in general corresponds a cubic in $\lambda$ and $\mu$ of the second degree at most in either $\lambda$ or $\mu$. If, however, 4 and 5 are chosen so that $\beta / \beta^{\prime}=\gamma / \gamma^{\prime}$, 
i.e., if they are in a line with 1 , then to a straight line corresponds a quadratic equation in $\lambda$ and $\mu$. For, if $\beta / \beta^{\prime}=\gamma / \gamma^{\prime}$

$$
\frac{1}{x}: \frac{1}{y}: \frac{1}{z}=\mathrm{L}: \mathbf{M}: \mathrm{N}
$$

where $\mathbf{L}, \mathbf{M}, \mathbf{N}$ are linear functions of $\lambda$ and $\mu$; and $\therefore$ to $\Sigma \mathrm{A} / y z=0$ corresponds

$$
\mathrm{AMN}+\mathrm{BNL}+\mathrm{CLM}=0 .
$$

Similarly if $2,3,4$ say are collinear, and also $1,2,5$ then the equations become

$$
\frac{\mathrm{P} 13}{\mathrm{P} 14}=c \lambda ; \frac{\mathrm{P} 13}{\mathrm{P} 23}=c^{\prime} \mu ;
$$

in which $c=23 / 24 ; c^{\prime}=15 / 25$. In such a case to a linear equation in $x$ and $y$ corresponds a linear equation in $\lambda$ and $\mu$ and inversely. We shall in general assume that no three points in question are collinear.

\$7. A number of interesting geometrical theorems may be more easily deduced by taking particular algebraic relations connecting $\lambda$ and $\mu$. In what follows, ordinary cartesian co-ordinates are used, and by $(\mathrm{Pab})$ is meant the determinant

$$
\left|\begin{array}{lll}
x & y & 1 \\
x_{a} & y_{a} & 1 \\
x_{b} & y_{b} & 1
\end{array}\right|
$$

Ex. 1. To $\lambda=c$ a constant corresponds the conic section given by

$$
\text { (P13) }(\mathrm{P} 24)=c(\mathrm{P} 14) \text { (P23.) }
$$

Ex. 2. To the relation

$$
\mathrm{A} \lambda+\mathrm{B} \mu=0
$$

corresponds likewise a conic through the points $1,2,4,5$ but not in general through 3 .

For

$$
\begin{aligned}
\mathrm{P}(1234) / \mathrm{P}(1235) & =\mathrm{P}(1254) . \\
\therefore \mathrm{P}(1254)=-\mathrm{B} / \mathrm{A} & =\text { constant }
\end{aligned}
$$

Ex. 3. To

$$
\mathrm{A} \lambda+\mathrm{B} \mu+\mathrm{C}=0
$$

corresponds $\mathrm{A} \times \mathrm{P}(1234)+\mathrm{B} \times \mathrm{P}(1235)+\mathrm{C}=0$,

i.e., $\mathrm{A}(\mathrm{P} 13)(\mathrm{P} 15)(\mathrm{P} 24)+\mathrm{B}(\mathrm{P} 13)(\mathrm{P} 14)(\mathrm{P} 25)+\mathrm{C}(\mathrm{P} 14)(\mathrm{P} 15)(\mathrm{P} 23)=0$ 
This equation in general represents a cubic curve possessing a double point at 1 , and ordinary points at $2,3,4,5$.

Now the datum of a double point is equivalent to three conditions, and each ordinary point is given by one condition. There are therefore a twofold infinity of cubics possessing a double point at 1 and through $2,3,4,5$. Also the equation in $\lambda$ and $\mu$ contains two arbitrary constants. We have, therefore, the following theorem suggested.

"Take a cubic with double point at 1 , and let. $2,3,4,5$ be any four fixed points on it. Let $\pi_{1}$ denote $P(1234)$, and $\kappa_{1}$ denote $P(1235), P$ being any other point on the cubic. There is a linear relation connecting $\pi_{1}$ and $\kappa_{1}$, and $\left(\pi_{1} \pi_{2} \pi_{3} \pi_{4}\right)=\left(\kappa_{3} \kappa_{1} \kappa_{3} \kappa_{4}\right)$."

For confirmation see Salmon's Higher Plane Curves, $\$ 163$.

Particular cases arise when 2 and 3 are the circular points at infinity.

But if $5(1234)=\alpha$, and $4(1235)=\beta$; and if $\mathrm{A} \alpha+\mathrm{B} \beta+\mathrm{C}=0$, then the curve given by $\mathrm{A} \lambda+\mathrm{B} \mu+\mathrm{C}=0$ is a degenerate cubic consisting of a conic and a straight line through 1 .

$E x$. 4. To the relation

$$
\mathrm{A} \lambda \mu+\mathrm{B} \lambda+\mathrm{C} \mu+\mathrm{D}=0
$$

corresponds

$$
\begin{gathered}
\mathrm{A}(\mathrm{P} 13)^{2}(\mathrm{P} 24)(\mathrm{P} 25)+\mathrm{B}(\mathrm{P} 13)(\mathrm{P} 23)(\mathrm{P} 15)(\mathrm{P} 24) \\
+\mathrm{C}(\mathrm{P} 13)(\mathrm{P} 23)(\mathrm{P} 14)(\mathrm{P} 25)+\mathrm{D}(\mathrm{P} 23)^{2}(\mathrm{P} 14)(\mathrm{P} 15)=0
\end{gathered}
$$

This equation represents a quartic curve in general, possessing double points at $1,2,3$, and ordinary points at 4,5 .

There are three arbitrary constants in the $\lambda-\mu$ equation. But only a threefold infinity of quartics are possible possessing nodes at three given points and through other two points. We have therefore the following theorem suggested.

"Take a tri-nodal quartic with nodes at $1,2,3$. Let 4 and 5 be any tyo fixed points on it, and $P$ an arbitrary point on the curve. Then $\left(\pi_{1} \pi_{2} \pi_{3} \pi_{4}\right)=\left(\kappa_{1} \kappa_{2} \kappa_{3} \kappa_{4}\right)$."

There are a variety of degenerate cases. For example if $A B C D$ are such that $A a \beta+B a+C \beta+D=0$, then the quartic reduces to the base conic 12345 , and a second conic through $1,2,3$, (Degenerate cases may also arise should any of the base points be collinear). 
Any relation $f(\lambda, \mu)=0$ will furnish a degenerate curre when $f(\alpha, \beta)=0$.

$E x$. 5. If a bilinear equation is given connecting $P(1234)$ and $P(5678)$ the locus of $P$ is in general a quartic; but in a large variety of cases the curve is of lower order.

(a.) If $\mathrm{P}(1342) / \mathrm{P}(1562)=\lambda$, a constant, the locus of $\mathrm{P}$ is a cubic (P14)(P32)(P56) $-\lambda(\mathrm{P} 34)(\mathrm{P} 16)(\mathrm{P} 52)=0$

passing through $1,2,3,4,5,6$; and through the intersection 7 of 14 and $52 ; 8$ of 32 and $16 ; 9$ of 56 and 34 . (Figure 8.)

The nine points thus obtained form nine associated points of a pencil if cubics in triads upon two systems of three lines; and one obtains ( $v$. Salmon's Higher Plane Curves) the most general form of the cubic, from which its more elementary properties are generally deduced. It will be noted that if 3,4 ; and 1,2 , are given on a fixed cubic, the points 5 and 6 are uniquely determined by the collinearities :-

$$
\begin{aligned}
& 147 \\
& 275
\end{aligned} \text { and } \begin{aligned}
& 238 \\
& 186 .
\end{aligned}
$$

Also 34 and 56 cut on the cubic.

The same cubic could be obtained by a variety of such equations, e.g., $\mathrm{P}(5164) / \mathrm{P}(5324)=\mu$, corresponding to

$$
\begin{aligned}
& 659 \\
& 943 \text { and } \quad 757 \\
&
\end{aligned}
$$

Since all the relations are algebraic, a bilinear equation, $\lambda=\mu$, connecting $\lambda$ and $\mu$ is suggested for the same cubic.$$
\mathbf{P}(2134) / \mathbf{P}(2536)=\lambda \text { gives }
$$$$
(\mathbf{P} 14)(\mathrm{P} 26)(\mathrm{P} 53)-\lambda(\mathrm{P} 56)(\mathbf{P} 24)(\mathbf{P} 13)=0 \text {, }
$$

a cubic through $1,2,3,4,5,6$ and through the intersections $7,8,9$ of 14 and $56 ; 26$ and $13 ; 53$ and 24 . (Figure 9.)

These again form nine associated points. They may also be found in any given cubic as follows.

Take the base points 1, 2, 5; and the point. 7. Form the Steinerian hexagon 
Then $P(2134) / P(2536)=$ constant for all points $P$ on the cubic.

Similiar base points for the same configuration are

$$
169: 237: 346: 458: 789 \text {. }
$$

Thus start with $1,(\because 7438$ excluded $)$. The other two base points must come from 2569 . Take 6 , thereby excluding 5728 , i.e., excluding in addition 5 and 2 , and leaving $9 ;. \therefore 169$ as possible base points

Thus

$$
\begin{aligned}
& 417 \\
& 765 \\
& 593 \\
& 318 \\
& 862 \\
& 294 ;
\end{aligned}
$$

and $\mathrm{P}(6137) / \mathrm{P}(6932)=\mu$ a constant.

Ex. 6. Consider the cross ratios

$$
1(2345): 2(3451) ; \ldots \ldots ; 5(1234) \text {. }
$$

Equate the product of two or more of these to a constant. Fix four of the points, when the fifth traces out a curve which is at most of the fourth degree, and is generally, but not always, unicursal.

(A) e.g. Let $1(2345) .2(3451) .3(4512)=$ constant.

(i) Put $\mathbf{P}$ for 5

$$
\begin{aligned}
& \therefore(\mathrm{P} 23)^{2} /(\mathrm{P} 12)(\mathrm{P} 24)=\text { constant } \\
& \therefore \text { the locus of } \mathrm{P} \text { is a pair of lines. }
\end{aligned}
$$

(ii) Put $P$ for 4 . The locus is again a degenerate conic.

(iii) Put $\mathbf{P}$ for 3

$\therefore(\mathrm{P} 15)(\mathrm{P} 25)(\mathrm{P} 23) /(\mathrm{P} 12)(\mathrm{P} 24)(\mathrm{P} 13)=$ constant.

The locus is a cubic with double point at 2 ; through 1 and 3 ; through the intersection of 15 and 24 , and of 25 and 13 ; and tangent to 15 at 1 .

(iv) Put $\mathrm{P}$ for 2

$$
\therefore(\mathrm{P} 14)^{2}(\mathrm{P} 35)^{2} /(\mathrm{P} 13)(\mathrm{P} 15)(\mathrm{P} 23)(\mathrm{P} 45)=\text { constant. }
$$

The locus is a quartic with double points at $1,3,5$, through 4 , but not through $2 ; 23$ is tangent where 14 again cuts the curve; 45 is tangent at 4.

(v) Put $\mathrm{P}$ for 1 when the locus is a pair of lines through 2. 
(B) Equate the product of the five ratios to a constant and put $\mathbf{P}$ for 5 , say.

$\therefore$ P13 . P23 . P24/P12 . P34 . P14= constant.

This is a cubic through $1,2,3,4: 13$ is tangent at 1,34 is tangent at 3,42 is tangent at 4 , and 21 is tangent at 2. Also 23 and 14 intersect on the curve.

The quadrilateral 1234 is therefore both inscribed and circumscribed to the cubic. A cubic curve with no double point has always a limited number of such quadrilaterals.

Ex. 7. $\mathrm{P}(1234) \times \mathrm{P}(1278)=$ constant

gives a quartic with double points at 1 and 2.

$$
\mathrm{P}(1234) \times \mathrm{P}(1678)=\text { constant }
$$

furnishes a quartic with a double point at 1 .

$$
\mathrm{P}(1234) \mathbf{P}(5678)=\text { constant }
$$

furnishes a quartic which does not in general possess a double point. 